\title{
Práticas de redução de massa corporal em judocas nos períodos pré-competitivos
}

CDD. 20.ed. 796.073

796.8

\author{
Sabrina Pinheiro FABRINI* \\ Ciro José BRITO*/** \\ Edmar Lacerda MENDES* \\ Cephora Maria SABARENSE* \\ João Carlos Bouzas MARINS* \\ Emerson FRANCHINI ${ }^{* * *}$
}

*Universidade Federal de Viçosa.

${ }^{* *}$ Centro Universitário Metodista de Minas Izabela Hendrix, Universidade Católica de Brasília.

${ }^{* * *}$ Escola de Educação Física e Esporte, Universidade de São Paulo.

\section{Resumo}

0 objetivo deste trabalho foi identificar, em judocas, os procedimentos adotados para a redução da massa corporal em períodos próximos às competições e discuti-los frente à literatura. Participaram deste estudo 105 do sexo masculino pertencentes às classes júnior e sênior (idade média de $21,5 \pm 8,0$ anos). De acordo com os resultados, $73,6 \%$ dos atletas desenvolveram estratégias de redução de massa corporal em períodos pré-competitivos, e os métodos mais utilizados por eles foram: aumento do número de atividades físicas além das habituais $(76,1 \%)$, restrição calórica $(59,8 \%)$, redução de carboidratos $(32,6 \%)$ e restrição de líquidos (20,7\%). Em valores absolutos, os judocas masculinos reduzem significativamente mais ( $p<$ $0,05)$ a massa corporal em relação às mulheres $(4,5 \pm 3,5$ e 1,7 $\pm 0,8 \mathrm{~kg}$, respectivamente). As mulheres iniciam com maior antecedência à competição $(p<0,05)$ a redução da massa corporal $(14 \pm 6$ e $19 \pm 7$ dias, respectivamente). Pode-se inferir que a maioria dos judocas adota estratégias de redução aguda de massa corporal em períodos que antecedem as competições, o que pode resultar na queda do rendimento.

UnItermos: Artes marciais; Treinamento desportivo; Perda de massa corporal; Desempenho atlético.

\section{Introdução}

As modalidades de luta apresentam a massa corporal como um dos critérios de divisão dos atletas. Em decorrência dessa categorização, muitos atletas optam por reduzir a massa corporal na tentativa de enfrentarem atletas menores e mais fracos (ARTIOLI, Franchini \& LANCHA JUNIOR, 2006). Contudo, na tentativa de adequação a uma categoria inferior à sua massa corporal, eles optam pela redução brusca (LEYDON \& Wall, 2002; Moore, Timperio, Crawford, Burns \& Cameron-Smith, 2002; Perriello, 2001). A perda de grande quantidade de massa corporal em menos de uma semana é a mais prejudicial ao organismo, ocasionando: redução de força muscular (FTAITI, Grélot, Coudreuse \& Nicol, 2001), declínio no tempo de desempenho (Cheung \& MCLellan, 1998), menor volume plasmático e sanguíneo (BARTHOLOMEW, O'Brien \& GILl, 2004), redução na eficiência do miocárdio (Mountain \& CoYle, 1992), diminuição do consumo máximo de oxigênio (MARINS, DANTAS $\&$ ZAMORRA-NAVARRO, 2000b), prejuízo ao processo termorregulatório (GonZÁlez-Alonso, Teller, Andersen, Jensen, Hyldig \& Nielsen, 1999), diminuição do fluido de sangue renal e do volume de líquidos filtrados pelo rim (MELIN, JIMENEZ, SAVOUREY, Bittel, Cottet-Emard, Pequignot, Allevard \& GHARIB, 1997), depleção dos estoques de glicogênio (Hargreaves, Dillo, Angus \& Febraio, 1995) e aumento de eletrólitos perdidos pelo corpo (AMERICAN College of SPORTS Medicine - ACSM, 1996).

Além de prejuízos fisiológicos, a perda rápida de massa corporal pode afetar a saúde mental do atleta, levando a problemas de concentração, memória e velocidade de processamento das informações, podendo causar até desordens alimentares (LANDERS, ARENT \& LuTZ, 2001). Considerando que uma luta de judô pode ser definida por uma técnica aplicada com perfeição 
(“Ippon"), a dificuldade de concentração e redução da capacidade de processamento de informações por parte do lutador pode aumentar a vantagem do adversário.

A desidratação forçada tem sido apresentada como o fator adverso mais significativo em relação à queda no desempenho. A perda de líquidos corporais induzida pela realização de exercícios em ambientes quentes e o uso de roupas antitranspirantes (borracha/ plástico), laxantes e diuréticos podem afetar o equilíbrio eletrolítico corporal, principalmente do mineral cálcio, o que pode resultar em menor mineralização óssea e causar fraturas por estresse (COHEN \& RoE, 2000). Frequentemente, os diuréticos produzem nos lutadores hipocalemia (diminuição da concentração de potássio no sangue), podendo colocar em risco a saúde, pois a redução do potássio corporal altera a

\section{Materiais e métodos}

Este estudo, realizado durante competições de judô e visitas a academias, caracteriza-se por ser transversal observacional. Antes do início, ele, foi aprovado pelo Comitê de Ética em Pesquisas com Seres Humanos da Universidade Federal de Viçosa, atendendo às orientações da Resolução 196/96 do CNS, de 10/10/96, sobre experimentos com seres humanos.

\section{Amostra}

Foram entrevistados 125 atletas, sendo 105 masculinos e 20 femininos. De acordo com as estatísticas da Federação Mineira de Judô (FMJ) e Liga Mineira de Judô (LMJ), existem 1.100 judocas masculinos e 225 femininos na faixa etária avaliada neste estudo. Assim, a amostra deste trabalho representa 9,5\% e $8,9 \%$ do total de judocas masculinos e femininos filiados a essas entidades, respectivamente. atividade da bomba sódio-potássio, a qual, em níveis críticos, leva o atleta a óbito (MARINS, DANTAS \& ZAMORRA-NAVARRO, 2000a).

A verificação da prevalência dos métodos de redução aguda de massa corporal pode auxiliar treinadores e nutricionistas no estabelecimento de estratégias de redução gradativa da massa corporal. Por meio desses resultados, é possível estabelecer campanhas educativas no sentido de banir esse tipo de prática do esporte, propiciando, assim, melhora no rendimento dos judocas, bem como proteção à saúde dos atletas. Diante do exposto, os objetivos deste trabalho foram: identificar em judocas os procedimentos de perda de massa corporal em períodos próximos a competições; e discutir os resultados em relação às recomendações de especialistas.

TABELA 1 - Distribuição da amostra em relação às categorias de peso do judô.

\begin{tabular}{lcccc}
\hline \multicolumn{1}{c}{ Categoria } & Limite de massa corporal & Homens & Limite de massa corporal & Mulheres \\
\hline Ligeiro & até $60 \mathrm{~kg}$ & 6 & até $48 \mathrm{~kg}$ & 2 \\
Meio leve & de 60 até $66 \mathrm{~kg}$ & 12 & de 48 até $52 \mathrm{~kg}$ & 3 \\
Leve & de 66 até $73 \mathrm{~kg}$ & 27 & de 52 até $57 \mathrm{~kg}$ & 5 \\
Meio médio & de 73 até $81 \mathrm{~kg}$ & 29 & de 57 até $63 \mathrm{~kg}$ & 6 \\
Médio & de 81 até $90 \mathrm{~kg}$ & 31 & de 63 até $70 \mathrm{~kg}$ & 4 \\
Meio pesado & de 90 até $100 \mathrm{~kg}$ & 0 & de 70 até $78 \mathrm{~kg}$ & 0 \\
Pesado & acima de $100 \mathrm{~kg}$ & 0 & acima de $78 \mathrm{~kg}$ & 0 \\
\hline
\end{tabular}




\section{Procedimentos}

Antes de iniciar a pesquisa, os judocas e senseis (professores) receberam informaçōes sobre o estudo, sua importância e foram consultados sobre seu consentimento em participar voluntariamente. Após a assinatura do termo de consentimento informado, os atletas foram entrevistados de maneira aleatória. Empregou-se metodologia exploratória, por meio de pesquisa do tipo descritiva, utilizando questionário padronizado com perguntas objetivas autoadministradas.

\section{Antropometria}

Mensuraram-se a estatura (ES) e a massa corporal (MC) em balança com estadiômetro acoplado (escala de $0,5 \mathrm{~cm}$ e resolução de $100 \mathrm{~g}$, respectivamente). $O$ percentual de gordura foi estimado indiretamente, pela técnica de dobras cutâneas. Para os homens, foram medidas as espessuras das dobras cutâneas triciptal, subescapular e abdominal. Quanto às mulheres, foram mensuradas as espessuras das dobras cutâneas triciptal e subescapular. As mensuraçōes seguiram os procedimentos propostos por LOHMAN (1992), usando um compasso Lange®.

No tocante à estimativa da densidade corporal em homens, foi utilizada a equação de THORLAND, Tipton, Lohman, Bowers, Housh, Jonhson, Kelly, Oppliger e Tcheng (1991); para as mulheres, estimou-se o percentual de gordura pela equação de Slaughter, lohman, Boileau, Horswill, StillMAN, VAN LoOn e BEMben (1988). O percentual de

\section{Resultados}

A TABELA 2 apresenta a idade, o tempo de experiência na modalidade e a caracterização antropométrica da amostra.

Observam-se na TABELA 2 diferenças antropométricas entre judocas masculinos e femininos no que se refere às variáveis: idade $(\mathrm{p}<0,05)$, experiência na modalidade, massa corporal, estatura e percentual de gordura $(\mathrm{p}<$ $0,01)$. No entanto, não houve diferença para a variável IMC $(p>0,05)$. A TABELA 3 descreve a proporção da amostra que se pesa antes e depois dos treinos, os judocas envolvidos em práticas de redução de massa corporal em períodos pré-competitivos, a perda máxima na semana pré-competição e o total da amostra que foi orientado sobre a redução ou o aumento da massa corporal. gordura, para homens, foi calculado pela equação de Brozek, Grande, Anderson e Keys (1963), pois ela foi adotada no estudo de ThORLAND et al. (1991).

Instrumento da pesquisa

Considerando o aspecto exploratório deste estudo, foi elaborado um questionário para tal, o qual foi validado por três professores que atuam nos Departamentos de Nutrição e Educação Física da Universidade Federal de Viçosa (ANEXO I).

\section{Análise estatística}

$\mathrm{Na}$ análise dos dados foi utilizada a distribuição percentual obtida em cada resposta, sendo descartadas as questóes não respondidas de cada questionário. $\mathrm{O}$ teste $\mathrm{t}$ foi utilizado para verificar diferenças significativas entre os sexos nas variáveis: idade, experiência na modalidade, massa corporal, estatura, IMC, percentual de gordura, idade na qual reduziu a massa corporal pela primeira vez, dias antes da competição que inicia a redução de massa corporal e perda de massa corporal na semana pré-competitiva. Utilizou-se ainda o teste do Quiquadrado a fim de verificar a existência de diferenças entre os sexos para as variáveis: peso antes e depois dos treinos, redução da massa corporal, e orientação em relação à manipulação da massa corporal, bem como para as variáveis relacionadas aos métodos de perda de massa corporal. O nível de significância adotado foi $5 \%$.
TABELA 2 - Idade, anos de experiência e características antropométricas dos judocas.

\begin{tabular}{|c|c|c|c|}
\hline Variável & Homens & Mulheres & \\
\hline $\mathrm{N}$ & 105 & 20 & $a=$ diferença entre os \\
\hline Idade & $24,7 \pm 2,1 \operatorname{anos}^{\mathrm{a}}$ & $21,2 \pm 1,5$ anos & grupos $(p<0,05)$. \\
\hline Experiência & $8,5 \pm 1,3 \operatorname{anos}^{\mathrm{a}}$ & $4,5 \pm 2,7$ anos & \\
\hline Massa corporal & $80,5 \pm 8,2 \mathrm{~kg}^{\mathrm{a}}$ & $58,5 \pm 4,9 \mathrm{~kg}$ & \\
\hline Estatura & $179,6 \pm 5,4 \mathrm{~cm}^{\mathrm{a}}$ & $157,2 \pm 7,4 \mathrm{~cm}$ & \\
\hline IMC & $24,5 \pm 2,5 \mathrm{~kg} / \mathrm{m}^{2}$ & $23,7 \pm 2,3 \mathrm{~kg} / \mathrm{m}^{2}$ & \\
\hline$\%$ de gordura & $16,9 \pm 3,2 \%{ }^{a}$ & $25,1 \pm 6,2 \%$ & \\
\hline
\end{tabular}


FABRINI, S.P. et al.

Em relação à TABELA 3, observou-se diferença significativa entre judocas masculinos e femininos para as variáveis: início de redução de massa corporal antes da competição e perda de massa corporal na semana pré-competitiva ( $\mathrm{p}<0,01$ para as duas variáveis). Não foram observadas diferenças entre as variáveis pesar-se antes e depois do treino, idade na qual reduziu a massa corporal pela primeira vez, redução de massa corporal e receber orientação em relação à manipulação da massa corporal ( $p$ > $0,05)$. Na TABELA 4 são apresentados os métodos utilizados pelos judocas avaliados para restrição da massa corporal.

De acordo com a TABELA 4, houve diferença significativa entre judocas masculinos e femininos no tocante às variáveis, aumento das atividades e dieta hipocalórica ( $\mathrm{p}<0,05$ para as duas comparaçōes). Não foram constatadas diferenças para as variáveis restrição de carboidratos, restrição de líquidos, e restrição de lipídios $(\mathrm{p}>0,05)$. Quanto às variáveis uso de diurético e/ou laxante e uso de sauna/roupas antitranspirantes, não foram obtidas respostas femininas. A FIGURA 1 apresenta a distribuição da amostra em relação às fontes nas quais os judocas obtiveram informaçôes quanto à manipulação da massa corporal.

Em relação à FIGURA 1, não foi realizada a separação entre sexo masculino e feminino, uma vez que em muitas variáveis as mulheres não tiveram representação devido ao pequeno tamanho da amostra.
* O valor entre parênteses representa 0 percentual de judocas que respondeu sim para esta questão.

${ }^{* *}$ Os valores entre parênteses representam 0 valor máximo e mínimo para esta variável. $\mathrm{a}=$ Diferença estatisticamente significativa $(\mathrm{p}$ $<0,05)$.

TABELA 3 - Características da amostra em relação ao controle da massa corporal, idade na qual reduziu a massa corporal, tempo pré-competição na qual inicia a redução, perda absoluta de massa corporal e orientação em relação à redução.

\begin{tabular}{lccc}
\hline \multicolumn{1}{c}{ Variável } & Homens & Mulheres & Amostra total \\
\hline Pesar-se antes e depois dos treinos* & $47(44,8 \%)$ & $12(60 \%)$ & $57(45,6 \%)$ \\
Idade na qual reduziu a massa corporal pela primeira vez (anos) & $17,4 \pm 1,5$ & $18,3 \pm 1,2$ & $17,6 \pm 1,3$ \\
Início, antes da competição, da redução de massa corporal* $^{*}$ & $14 \pm 6$ & $19 \pm 7^{\text {a }}$ & $16 \pm 7$ \\
Reduz a massa corporal & $81(77,1 \%)$ & $11(55,0 \%)$ & $92(73,6 \%)$ \\
Perda na semana pré-competitiva $(\mathrm{kg})^{* *}$ & $4,5 \pm 3,5(1-10)$ & $1,7 \pm 0,8(1-3)^{\mathrm{a}}$ & $4,0 \pm 3,1(1-10)$ \\
Recebe orientação em relação à manipulação da massa corporal $^{*}$ & $85(81 \%)$ & $15(75 \%)$ & $100(80 \%)$ \\
\hline
\end{tabular}

TABELA 4 - Métodos utillizados por judocas para redução da massa coporal.

\begin{tabular}{|c|c|c|c|c|}
\hline \multirow{8}{*}{$\begin{array}{l}a=\text { Diferença estatisti- } \\
\text { camente significativa }(p \\
<0,05) .\end{array}$} & Método & Homens & Mulheres & Total \\
\hline & Aumento de atividades & $64(79,05 \%)$ & $6(54,6 \%)^{a}$ & $70(76,1 \%)$ \\
\hline & Dieta hipocalórica & $51(63,0 \%)$ & $4(36,4 \%)^{a}$ & $55(59,8 \%)$ \\
\hline & Restrição de carboidratos & $26(32,1 \%)$ & $4(36,4 \%)$ & $30(32,6 \%)$ \\
\hline & Restrição de líquidos & $17(21,0 \%)$ & $2(18,2 \%)$ & $19(20,7 \%)$ \\
\hline & Restrição de lipídios & $10(12,4 \%)$ & $1(9,1 \%)$ & $11(12,0 \%)$ \\
\hline & Uso de diurético e/ou laxante & $10(12,4 \%)$ & 0 & $10(10,9 \%)$ \\
\hline & Uso de sauna/roupas antitranspirantes & $9(11,1 \%)$ & 0 & $9(9,8 \%)$ \\
\hline
\end{tabular}

168 • Rev. bras. Educ. Fís. Esporte, São Paulo, v.24, n.2, p.165-77, abr./jun. 2010 


\section{Orientação relacionada à manipulação da massa corporal}

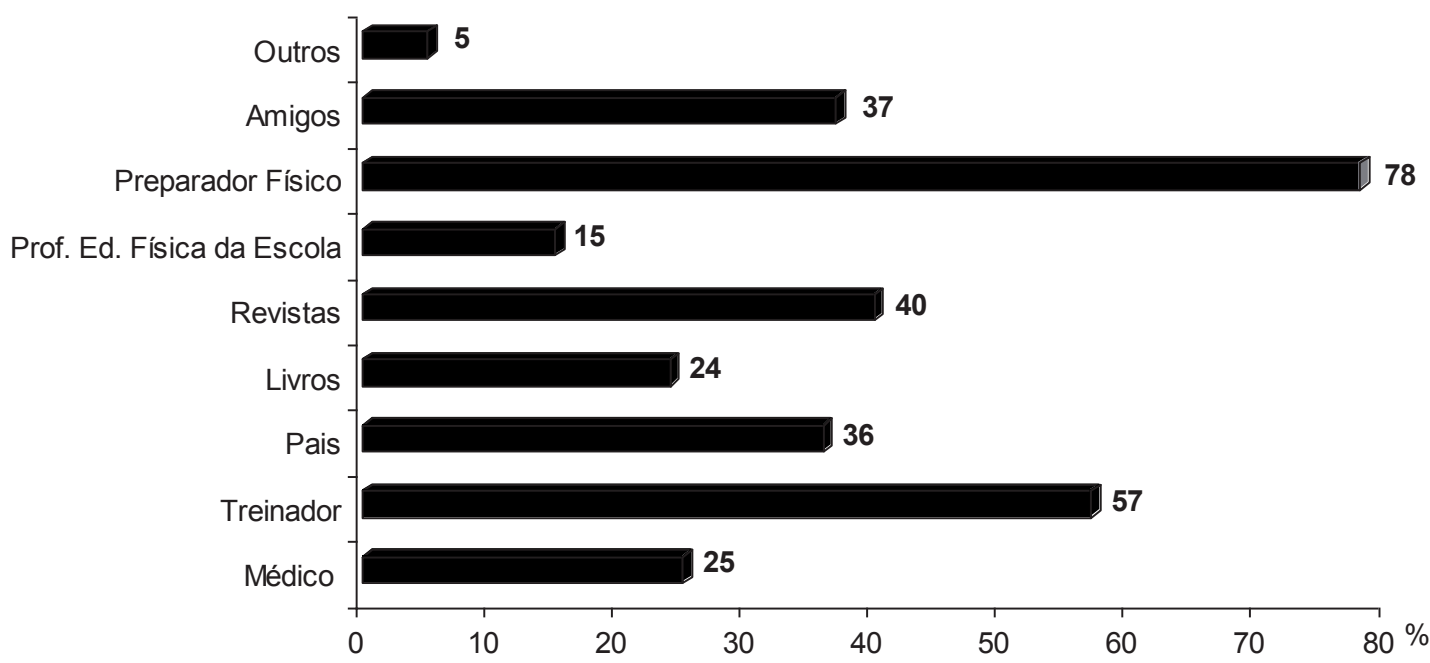

FIGURA 1 - Distribuição da amostra em relação à fonte na qual obteve orientação para manipular a massa corporal.

\section{Discussão}

Entre os principais resultados deste estudo, observou-se que a maioria dos judocas entrevistados já realizou redução de massa corporal aguda, sendo o aumento das atividades físicas a principal estratégia utilizada. Um fator preocupante foi a idade na qual se utilizou pela primeira vez a redução brusca de massa corporal (aproximadamente 17 anos). Os principais orientadores, em relação à manipulação da massa corporal, são o preparador físico e os treinadores.

O percentual de judocas que adotavam estratégias para redução de massa foi superior ao encontrado por STEEN e BRownell (1990), os quais reportaram que $41 \%$ de 431 lutadores adotavam estratégias agudas de redução de massa corporal. Resultados semelhantes aos desses autores foram obtidos por KININGHAM e GORENFLO (2001), ao registrarem prevalência de $40 \%$ em 2.532 lutadores. Esses resultados indicam que os homens utilizam a redução da massa corporal com maior incidência que o relatado pelo ACSM (1996) em lutadores norte-americanos, cuja prevalência ficou entre 25 e $67 \%$. Já os resultados entre as mulheres estão na faixa esperada (ACSM, 1996). Contudo, os valores deste estudo são ligeiramente inferiores ao reportado em judocas avaliados por ARTIOLI (2008), ou seja, 86\%, em estudo com 790 atletas de judô $(592$ do sexo masculino e 198 do sexo feminino), dos quais 16,7\% tinham nível regional; 38,1\% nível estadual;
23,7\% nível nacional; e 21,5\% nível internacional. Esses dados apontam que os judocas deste estudo apresentam prevalência de estratégias de perda de massa corporal extremamente elevada em relação a outros grupos de atletas de luta, porém similares ao reportado em levantamento recente, realizado em outro Estado brasileiro, com atletas de judô.

De acordo com o observado na TABELA 2, tanto a amostra do sexo masculino quanto a do sexo feminino podem reduzir a massa corporal para competirem em categorias de peso inferiores, tendo em vista que apresentam percentual de gordura de $16,9 \pm 3,1 \%$ e $25,1 \pm 6,1 \%$, respectivamente. Segundo Perriello (2001), lutadores homens podem apresentar no mínimo de $7 \%$ de gordura corporal sem prejudicar a saúde; já para mulheres o limite inferior seria de $12 \%$. Assim, um planejamento nutricional realizado durante a preparação para a competição auxiliaria esses lutadores a reduzirem a massa corporal sem o uso de métodos de rápida redução da massa corporal. No entanto, não parece ser essa a realidade da amostra avaliada, uma vez que $77,1 \%$ dos homens e $55 \%$ das mulheres relataram reduzir a massa corporal na semana pré-competitiva, esses dados são similares aos reportados por ARTIOLI (2008), que observou média de $7 \pm 7$ dias antes da competição para a perda de peso. 
Entre os métodos utilizados, têm-se a sauna, o treinamento em lugares abafados e o uso de roupas impermeáveis, laxantes e diuréticos (STEEN \& BROWNELL, 1990). No presente estudo também foram identificadas essas açôes como forma de controle da massa corporal. A manipulação inadequada da massa corporal não causa prejuízos apenas no desempenho, mas coloca em risco também a vida do atleta (CLARK, 1997). De acordo com o Centro Americano para Controle e Prevenção de Doenças, três atletas faleceram em 1997, tentando reduzir a massa corporal em período pré-competitivo (CENTERS FOR DISEASE Control and Prevention - CDC, 1998).

Neste trabalho, foi relatado por alguns atletas que a adoção de estratégias de rápida perda de massa corporal teve início quando eles apresentavam aproximadamente 17 anos de idade. A adoção desse tipo de estratégia tão precocemente pode causar prejuízos ao desenvolvimento corporal do adolescente; segundo RoEMMICH e SinNING (1997), a desidratação forçada leva à menor liberação do hormônio do crescimento, fator de crescimento ligado à insulina-I e testosterona em lutadores. Atletas em fase de crescimento podem prejudicar o seu desenvolvimento corporal utilizando esses procedimentos inadequados, embora haja ajuste compensatório no processo no período pós-temporada.

BENARDOT (2001) observou que perdas agudas de massa corporal também podem estar relacionadas a transtornos psicológicos, existindo ainda evidências que apontam para prejuízo na memória de curto prazo, podendo influir em resultados acadêmicos ou em informaçôes táticas passadas pelo treinador entre uma luta e outra durante a competição.

Apesar de os indicadores de comprometimento fisiológico associados à desidratação estarem bem descritos, o trabalho de Smith, Dyson, Hale, Hamilton, Kelly e Wellington (2001) com boxeadores foi surpreendente. Os autores provocaram uma situação de restrição hídrica de até um litro e calórica em até $1.000 \mathrm{kcal}$ por dia, durante cinco dias, em oito lutadores, ocasionando, ao final do período, perda de $3 \%$ da massa corporal. Os resultados mostraram não haver diferenças na frequência cardíaca e na potência de golpes, indicando que a restrição hídrica e a calórica, quando controladas, podem não trazer prejuízos aos atletas. Da mesma forma, entre atletas de judô habituados à redução de massa corporal, foi observado que $5 \%$ de diminuição em uma semana não resultou em queda do desempenho em três testes de Wingate para membros superiores, com intervalos de três minutos entre eles (ArTiOLI, 2008).
O aumento das atividades objetivando a redução de massa corporal, que foi o método de redução da massa corporal mais prevalente no presente estudo, é uma estratégia que pode ser recomendada, desde que realizada em períodos de preparação, uma vez que a recuperação da homeostasia dos líquidos pode levar de 24 a 48 horas; a do glicogênio muscular, até 72 horas; e a de outros tecidos magros, em períodos ainda maiores (ACSM, 1996). Um período de 24 horas não foi suficiente para a completa reidratação após redução de $6 \%$ da massa corporal em judocas e lutadores (FoGELHOLM, Koshinen, LaAkso, Rakinen \& Ruokonen, 1993).

$\mathrm{O}$ aumento na quantidade de exercícios físicos, principalmente de caráter aeróbio, deve estimular a mobilização de gordura e produzir gasto energético ainda maior, viabilizando assim a redução do percentual de gordura sem comprometimento da massa corporal magra (Oppliger, Nelson SteEn \& SCOtT, 2003). Moore et al. (2002) observaram essa estratégia em jóqueis australianos. Já em judocas espanhóis, DiAZ (2003) registrou valores bem inferiores aos obtidos no presente estudo em $44 \%$ de atletas que utilizavam aumento da carga de atividade para reduzir a massa corporal. Sobre esse tipo de estratégia, Oppliger, Nelson Steen e Scott (2003) constataram que era o segundo método na preferência dos lutadores americanos, com prevalência de 75,2\%.

No presente estudo, os resultados indicaram que a dieta hipocalórica foi adotada por aproximadamente $60 \%$ dos entrevistados (TABELA 4), representando a segunda estratégia de perda de massa corporal adotada pelos judocas. Na tentativa de atingir a massa corporal inferior, a diminuição calórica diária ocorre pela ação de eliminar uma refeição do dia, como observado em $75 \%$ dos atletas $(\mathrm{n}=116)$ entrevistados por Moore et al. (2002).

A restrição alimentar é usualmente empregada em modalidades que são categorizadas por peso, havendo registros de ausência total de consumo energético por até dois dias antes da competição (Benardot, 2001). No presente estudo, a prevalência de restrição calórica é inferior à reportada por Diaz (2003), o qual observou que $71,4 \%$ dos judocas e lutadores buscavam, através dessa estratégia, reduzir a massa corporal. É importante considerar que o suprimento calórico diário inferior a $1.500 \mathrm{kcal}$, de forma contínua, está relacionado ao consumo inadequado de vitaminas e proteínas (Clark, 1997). A redução no consumo de vitaminas pode afetar o desempenho do atleta em longo prazo, visto que essas substâncias, além de desempenharem funções metabólicas, atenuam o estresse oxidativo 
pelo combate aos radicais livres, devido à sua ação antioxidante (Aguiló, Tauler, Fuentespina, Tur, Córdova \& Pons, 2005; Mastaloudis, Morrow, Hopkins, Devaraj \& Traber, 2004).

Às vésperas da competição não é recomendada a restrição alimentar aguda (ACSM, 1996). A nutrição adequada pode otimizar as reservas de carboidratos (glicogênio muscular e hepático) para a competição, o que pode representar importante diferença no desempenho tanto em atividades de velocidade quanto de resistência. Durante o exercício de alta intensidade, Carter, Pringle, Boobis, Jones e Doust (2004) observaram que a depleção do glicogênio afeta a capacidade de resistência, devido à redução da captação de oxigênio. Por sua vez, o consumo de carboidratos pode atenuar a perda de força e o aparecimento da fadiga central e periférica (NyBO, 2003).

Apesar de a restrição de carboidratos ser utilizada por aproximadamente 33\% dos entrevistados, ela não é recomendada em períodos pré-competitivos, pois o glicogênio depletado durante os treinos deve ser recuperado através de dieta com alto teor de carboidratos (Van Hall, ShirrefFs \& Calbet, 2000). Além disso, os carboidratos atuam como o nutriente energético para a atividade anaeróbia (Arkinstall, Bruce, Clark, Rickards, Burke \& HaWley, 2004), que é a característica predominante das ações decisivas de esportes de combate, como o judô (Franchini, 2001). Segundo Kowatari, Umeda, Shimoyama, Nakaji, Yamamoto e Sugawara (2001), a redução de massa corporal em judocas, associada à baixa ingestão de carboidratos, resulta em menor atividade do sistema imune, aumentando a propensão do atleta a infecções.

A restrição de líquidos, utilizada por $20,2 \%$ dos atletas, também representa uma estratégia de redução de massa corporal inadequada. A combinação entre restrição alimentar e líquidos apresenta efeito fisiológico potencializador, que deixa o lutador despreparado para competir (ACSM, 1996). A diminuição do consumo de líquidos representa uma ação frequente nas modalidades em que a massa corporal é determinante (BERNADOT, 2001; BRITO \& Marins, 2005; Clark, 1997; Diaz, 2003). No tocante à relação entre desidratação e desempenho, Marins, Dantas e Zamorra-Navarro (2000b) publicaram uma revisão em que são apontadas 21 alterações fisiológicas prejudiciais ao desempenho decorrentes da desidratação. De acordo com BRITO (2005), o treinamento de judô tem duração de aproximadamente duas horas, e a restrição de líquidos durante toda a atividade pode provocar prejuízos fisiológicos proporcionais à perda hídrica (MARINS, DANTAS \& ZAMORRA-NAVARRO, 2000b).

Para reduzir os riscos de efeitos adversos para a saúde e o desempenho, a perda de massa corporal não deve exceder 1 a $2 \%$ da massa corporal por semana (AMERICAN Dietetic Association - ADA. ACSM. Dietitians of CANADA - DA, 2000). Neste estudo foi relatada perda máxima de $10 \mathrm{~kg}$ em uma semana (TABELA 3), o que representava $13,2 \%$ da massa corporal daquele atleta.

Deve-se ressaltar que, na amostra avaliada, apenas três lutadores relataram redução superior a $10 \mathrm{~kg}$ de massa corporal, porém essa prática inadequada pode ser observada com maior frequência em outros estudos (Kinningham \& Gorenflo, 2001; STEEN \& BROWNell, 1990). Oppliger, NeLSON-STEEN e Scott (2003) verificaram que $17,6 \%$ dos atletas apresentaram redução superior a $6,8 \mathrm{~kg}$ antes da pesagem da competição.

A restrição de lipídios, apesar de utilizada por apenas $17,4 \%$, pode ser adequada e recomendada quando a perda de massa corporal for almejada e planejada em longo período. No entanto, devese ressaltar que um nutricionista deve planejar a restrição de gorduras, uma vez que existem ácidos graxos que são essenciais e, portanto, não devem ser retirados da dieta (ADA. ACSM. DA, 2000).

Recomenda-se, para redução saudável da massa corporal, que a redução de gorduras não ultrapasse $15 \%$ do total energético consumido. A ausência delas na dieta pode afetar a saúde do atleta. Além disso, os lipídios são fontes de vitaminas lipossolúveis. Antes de se iniciar um programa de redução da massa corporal, é necessária a avaliação da composição corporal para verificar se há possibilidade ou não de reduzir (CLARK, 1997).

Segundo o ACSM (1996), lutadores, em geral, apresentam em média de 6 a $7 \%$ de gordura. Indivíduos nessa faixa não apresentam possibilidade de redução da massa, pois estão próximos do limite recomendado para judocas (FranCHINI, 2001). Caso um lutador, nessas condições, adote alguma estratégia de perda de massa corporal, esta será obtida pela redução da massa magra e perda de líquidos corporais. Conforme as regras do National College Athletic Association (NCAA), lutadores não podem competir com percentual de gordura inferior a $5 \%$, medido por pesagem hidrostática ou por equações de dobras cutâneas (NCAA, 2005) (as mesmas equações foram adotadas neste estudo). No presente estudo (TABELA 2), os registros de percentual de gordura apontam que boa parte de atletas tem condições de reduzir a massa corporal sem comprometer a massa corporal magra. Essa estratégia deve ser planejada 
adequadamente para que o atleta não tenha prejuízo em sua capacidade de rendimento físico.

Do total de entrevistados, apenas $10,9 \%$ utilizaram laxantes ou diuréticos para promover a perda da massa corporal. Esses valores são superiores aos registrados por DiAZ (2003), que observou prevalência de 5,8\%. O uso de diuréticos traz diversos efeitos adversos ao desportista, visto que a maior parte de líquidos perdida irá afetar tanto a termorregulação quanto a função cardiovascular (Marins, Dantas \& Zamorra-Navarro, 2000a).

Os diuréticos podem afetar também a função neuromuscular (SAWKa \& MONTAIN, 2000), reduzir o desempenho, provocar cãibras e produzir hipotensão arterial excessiva, tontura, desmaio, anorexia, vômito, distensão abdominal, ausência de reflexos e depressão mental (MARINS, DANTAS \& ZAMORRA-NAVARRO, 2000b). A utilização de vômito e diarréia induzidos causa também perda excessiva de minerais, fraqueza muscular e deterioração da função neuromuscular (VANIN \& SAYLOR, 1989). Outro agravante no uso de diuréticos é o fato de esse elemento fazer parte da lista de agentes dopantes da Agência Mundial Anti-Doping (World AnTIDoping Agency - WADA, 2005); nesse caso, o atleta poderá ser punido, caso haja um exame.

A frequência acumulada do percentual de atletas que adotaram medidas inadequadas para redução de massa corporal através da eliminação de líquidos - isto é, uso de roupas antitranspirantes, sauna, diuréticos e laxantes - foi de 19,7\%. No estudo de Oppliger, Nelson-Steen e Scott (2003), com lutadores colegiais e universitários norte-americanos, $25 \%$ dos atletas afirmaram utilizar métodos de perda rápida, como o uso de roupas antitranspirantes, jejum, laxantes, diuréticos e sauna. Em judocas e lutadores espanhóis, a prevalência de perda aguda de massa corporal através de sauna foi de $13 \%$, enquanto o uso de roupa plástica representou 14,5\% (DiAZ, 2003). Já em lutadores norte-americanos esses valores foram de $27,6 \%$, através da sauna, e 26,7\%, com roupas plásticas (Oppliger, NelsonSTEEN \& ScotT, 2003). Esses resultados, em seu conjunto, reforçam a ideia de que a perda hídrica aguda por parte dos atletas é frequente, colocando em risco a integridade física do lutador.

$\mathrm{O}$ uso de roupas antitranspirantes prejudica a dissipação do calor corporal, o que resulta em aumento da temperatura interna. A elevação da temperatura interna poderá causar cãibras, fraqueza generalizada, fadiga, náuseas e diarréia (MARINS, DANTAS \& ZAMORRA-NAVARro, 2000b). A utilização da sauna diminui os estoques corporais de água, resultando em hemoconcentração, o que pode contribuir para que o lactato se acumule mais rapidamente em exercício subsequente (Cheung \& McLellan, 1998). A NCAA e a National Athletic Trainers' Association (NATA) proíbem de competir atletas que fizerem uso de sauna em períodos que antecedem as competições (Casa, Armstrong, Hillman, Mountain, Reiff, Rich, ROBERTS \& STONe, 2000; NCAA, 2005).

Além da intensidade imposta pelo treinamento, o judoca está sujeito ao estresse térmico elevado devido ao uso do judogui, um equipamento que não pode ser eliminado da prática do esporte (BRITO \& MARINS, 2005). Somar ao estresse da modalidade métodos inadequados de perda de massa corporal por desidratação pode agravar ainda mais os efeitos fisiológicos que prejudicam o desempenho e a saúde do atleta (BRito, 2005).

A fim de banir do esporte tais práticas, é preciso estabelecer campanhas periódicas para o esclarecimento de atletas e treinadores sobre os perigos da perda rápida de massa corporal. A participação do nutricionista no planejamento da perda de massa corporal poderá contribuir de forma decisiva para escolhas dietéticas adequadas. As entidades responsáveis pelo esporte devem estabelecer regras que coíbam a utilização desses procedimentos por lutadores, assim como as estabelecidas pela NCAA.

Assim como neste estudo, as entidades responsáveis pelo esporte deveriam mapear a utilização desses procedimentos inadequados e estabelecer regras que coíbam essas práticas inadequadas. Após a morte de três lutadores nos Estados Unidos, a NCAA estabeleceu regras para coibir a perda aguda. De acordo com o estudo de Oppliger, Nelson-Steen e Scott (2003), 40\% dos atletas afirmaram que essas novas regras dificultam a utilização de procedimentos inadequados. O estabelecimento do percentual mínimo de gordura (5\%) foi uma das regras implantadas, a qual poderia ser adotada também pela Confederação Brasileira de Judô (CBJ), pela Liga Brasileira de Judô (LBJ) e pela Federação Internacional de Judô (FIJ), uma vez que essas entidades não estabelecem nenhum tipo de ação que coíba a utilização de estratégias para redução brusca da massa corporal.

Juntamente com a atualização do regulamento, as entidades devem estabelecer campanhas para orientaçôes de treinadores, visto que o conhecimento dos treinadores se reflete nas orientações que eles passam aos atletas (Perriello, 2001; RockWell, NickolsRichARDSON \& THYE, 2001). Essas ações são fundamentais, tendo em vista que os treinadores nem sempre possuem informações adequadas, como ficou evidenciado no trabalho de Sossin, GizIs, MarquarT e Sobal 
(1997) sobre nível de conhecimento, opinião e atitudes de treinadores americanos de luta. Outro exemplo é o estudo de JuZWIAK e ANCONA-Lopez (2004), no qual ficou demonstrado o desconhecimento dos treinadores acerca da perda de massa corporal; $8 \%$ dos treinadores indicavam aos seus atletas o uso de sauna com mais de quatro horas de duração, ao passo que 19\% recomendavam exercícios com roupas plásticas.

Recomenda-se que os atletas avaliem sua composição corporal, incluindo a proporcionalidade entre gordura e massa magra, antes de iniciar a perda de massa corporal. Caso o atleta apresente massa de gordura reduzida (até 5\%), ele deve ser desencorajado a reduzir a massa corporal. A perda de massa corporal deve ser gradual, fora dos períodos de competição, utilizando atividade física e dieta bem planejada; deve-se também evitar e desencorajar a utilização de métodos para perda rápida.

Cabe destacar que é necessária uma análise mais crítica dos resultados obtidos entre as lutadoras. Uma amostra maior aumentaria a gama de respostas, enriquecendo assim a discussão do trabalho. Nota-se a carência de trabalhos relacionados à área no Brasil, uma vez que alguns dos atletas avaliados apresentam resultados expressivos em competições internacionais. Estudos realizados com judocas brasileiros poderiam auxiliar os treinadores no planejamento de suas atividades (BRITO \& MARINS, 2005).

\title{
Considerações finais
}

De acordo com as respostas obtidas, conclui-se que a maioria dos atletas avaliados adota estratégias de redução de massa corporal de forma aguda, sendo empregadas ações não recomendadas, as quais podem prejudicar o rendimento ou a saúde do lutador. $\mathrm{O}$ aumento das atividades habituais é o procedimento mais adequado; entretanto, deve ser realizado durante o período de preparação e não nos momentos que antecedem as competiçôes.

\begin{abstract}
Practices for reducing body mass of judokas in the pre-competitive season

The aim of this work was to evaluate the procedures adopted for weight loss in periods close to competitions, and emphasize their physiologic effects. One hundred and twenty-five athletes (20 female and 105 male) belonging to the Juvenil, Junior and Senior categories $(21.5 \pm 8.0$ years old $)$ participated in this study. According to the results, $73.6 \%$ of the athletes used strategies of weight reduction methods during the pre-competitive periods. The methods used by the athletes were: increasing physical activity level $(76.1 \%)$, caloric restriction (59.8\%), restriction of carbohydrates (32.6\%), and restriction of liquids $(20.7 \%)$. In absolute values, male Judokas presented greater reductions in $(p<0.05)$ body mass compared to women $(4.5 \pm 3.5$ and $1.7 \pm 0.8 \mathrm{~kg}$, respectively). Women started using pre-competition strategies $(p<0.05)$ to reduc body weight sooner than men $(14 \pm 6$ and $19 \pm 7$ days, respectively). In conclusion, the most of judokas develop strategies for acute body mass reduction in pre-competitive periods, which may decrease their performance.
\end{abstract}

UnITERMS: Martial arts; Training; Weight loss; Athletic performance.

\section{Referências}

AGUILÓ, A.; TAULER, P.; FUENTESPINA, E.; TUR, J.Á.; CÓRDOVA, A.; PONS, A. Antioxidant response to oxidative stress induced by exhaustive exercise. Physiology and Behavior, London, v.84, n.1, p.1-7, 2005. AMERICAN COLLEGE OF SPORTS MEDICINE (ACSM). Position stand: weight loss in wrestlers. Medicine Science in Sports and Exercise, Madison, v.28, n.6, p.ix-xii, 1996. 
AMERICAN DIETETIC ASSOCIATION (ADA). AMERICAN COLLEGE OF SPORTS MEDICINE (ACSM). DIETITIANS OF CANADA (DA). Joint position statement: nutrition and athletic performance. Medicine Science in Sports and Exercise, Madison, v.32, n.12, p.2130-45, 2000.

ARKINSTALL, M.J.; BRUCE, C.R.; CLARK, S.A.; RICKARDS, C.A.; BURKE, L.M.; HAWLEY, J.A. Regulation of fuel metabolism by preexercise muscle glycogen content and exercise intensity. Journal of Applied Physiology, Washington, v.97, n.6, p.2275-83, 2004.

ARTIOLI, G.G. Estudo sobre perda rápida de peso no judô: prevalência, magnitude, métodos e efeitos sobre o desempenho. 2008. 104f. Dissertação (Mestrado em Educação Física) - Universidade de São Paulo, São Paulo, 2008.

ARTIOLI, G.G.; FRANCHINI, E.; LANCHA JUNIOR, A.H. Perda rápida de peso em esportes de combate de domínio: revisão e recomendações aplicadas. Revista Brasileira de Cineantropometria e Desempenho Humano, Florianópolis, v.8, n.2, p.92-101, 2006.

BARTHOLOMEW, K.; O’BRIEN, B.J.; GILL, N.D. Exercise-induced hypervolemia maybe not be consequential to dehydration during exercise. Journal of Sports Science and Medicine, Bursa, v.3, p.50-5, 2004. Young Investigator Special Issue. BENARDOT, D. Nutrición para deportistas de alto nivel. Barcelona: Hispano-Europea, 2001.

BRITO, C.J. Hidratação com e sem carboidratos durante o treinamento de judô. 2008. 100f. Dissertação (Mestrado em Ciência da Nutrição) - Universidade Federal de Viçosa, Viçosa, 2005.

BRITO, C.J.; MARINS, J.C.B. Caracterização das práticas de hidratação em judocas do estado de Minas Gerais. Revista Brasileira de Ciência e Movimento, Brasília, v.13, n.2, p.59-74, 2005.

BROZEK, J.; GRANDE, F.; ANDERSON, J.T.; KEYS, A. Densitometric analysis of body composition: Revision of some quantitative assumptions. Annals of the New York Academy of Sciences, New York, v.110, p.113-40, 1963.

CARTER, H.; PRINGLE, J.S.M.; BOOBIS, L.; JONES, A.M.; DOUST, J.H. Muscle glycogen depletion alters oxygen uptake kinetics during heavy exercise. Medicine Science in Sports and Exercise, Madison, v.36, n.6, p.965-72, 2004.

CASA, D.J.; ARMSTRONG, L.E.; HILLMAN, S.K.; MONTAIN, S.J.; REIFF, R.V.; RICH, B.S.E.; ROBERTS, W.O.; STONE, J.A. National Athletic Trainers Association Position Statement: fluid replacement for athlete. Journal of Athletic Training, Dallas, v.35, n.2, p.212-24, 2000.

CENTERS FOR DISEASE CONTROL AND PREVENTION (CDC). Hyperthermia and dehydration-related deaths associated with intentional rapid weight loss in three collegiate wrestlers-North Carolina, Wisconsin, and Michigan, November-December 1997. The Journal of the American Medical Association, Atlanta, v.279, n.11, p.824-5, 1998.

CHEUNG, S.S.; McLELLAN, T.M. Influence of short-term aerobic training and hydration status on tolerance during uncompensable heat stress. European Journal Applied Physiology and Occupational Physiology, Berlin, v.78, n.1, p.50-8, 1998. CLARK, N. Nancy Clark's sports nutrition guidebook. Champaign: Human Kinetics, 1997.

COHEN, A.J.; ROE, F.J.C. Review of risk factors for osteoporosis with particular reference to a possible aetiological role of dietary salt. Food and Chemical Toxicology, Oxford, v.38, n.2-3, p.237-53, 2000.

DIAZ, J. Trastornos de alimentación en el deporte. Sevilla: Wancevlen, 2003.

FOGELHOLM, G.M.; KOSKINEN, R.; LAAKSO, J.; RAKINEN, T.; RUOKONEN, I. Gradual and rapid weight loss: effects on nutrition and performance in male athletes, Medicine Science in Sports and Exercise, Madison. v.25, n.3, p.371-7, 1993. FRANCHINI, E. Judô: desempenho competitivo. São Paulo: Manole, 2001.

FTAITI, F.; GRÉLOT, L.; COUDREUSE, J.M.; NICOL, C. Combined effect of heat stress, dehydration and exercise on neuromuscular function in humans. European Journal Applied Physiology, Berlin, v.84, n.1-2, p.87-94, 2001.

GONZÁLEZ-ALONSO, J.; TELLER, C.; ANDERSEN, S.L.; JENSEN, J.B.; HYLDIG, T.; NIELSEN, B. Influence of body temperature on the development of fatigue during prolonged exercise in the heat. Journal of Applied Physiology, Washington, v.86, n.3, p.1032-9, 1999.

HARGREAVES, M.; DILLO, P.; ANGUS, D.; FEBRAIO, M. Effect of fluid ingestion on muscle metabolism during prolonged exercise. Journal of Applied Physiology, Washington, v.80, n.1, p.363-6, 1995.

JUZWIAK, C.; ANCONA-LOPEZ, F. Evaluation of nutrition knowledge and dietary recommendation by coaches of adolescent Brazilian athletes. International Journal of Sport Nutrition and Exercise Metabolism, Champaign, v.14, n.2, p.222-35, 2004.

KINNINGHAM, R.B.; GORENFLO, D.W. Weight loss methods in high school wrestlers. Medicine Science in Sports and Exercise, Madison, v.33, n.5, p.810-3, 2001.

KOWATARI, K.; UMEDA, T.; SHIMOYAMA, T.; NAKAJI, S.; YAMAMOTO, Y.; SUGAWARA, K. Exercise training and energy restriction decrease neutrophil phagocytic activity in judoists. Medicine Science in Sports and Exercise, Madison, v.33, n.4, p.519-24, 2001. 
LANDERS, D.M.; ARENT, S.M.; LUTZ, R.F Affect and cognitive performance in high school wrestlers undergoing rapid weight loss. Journal of Sports \& Exercise Psychology, Champaign, v.23, n.4, p.307-16, 2001.

LEYDON, M.; WALL, C. New Zealand jockeys dietary habits and their potential impact on health. International Journal of Sport Nutrition and Exercise Metabolism, Champaign. v.12, n.2, p.220-37, 2002.

LOHMAN, T.G. Advances in body composition assessment. Champaign: Human Kinetics, 1992.

MARINS, J.C.B.; DANTAS, E.H.M.; ZAMORRA-NAVARRO, S. Variaciones del sodio y potasio plasmáticos durante el ejercicio físico: factores asociados. Apunts: Educación Física y Deportes, Barcelona, v.9, n.62, p.48-55, 2000a.

Deshidratación y ejercicio físico. Selección: Revista Española de la Educación Física y el Deporte, Madrid, v.9, n.3, p.149-63, 2000b.

MASTALOUDIS, A.; MORROW, J.D.; HOPKINS, D.W.; DEVARAJ, S.; TRABER, M.G. Antioxidants supplementation prevents exercise-induced lipid peroxidation, but no inflammation, in ultramarathon runners. Free Radical Biology $\&$ Medicine, New York, v.36, n.10, p.1329-41, 2004.

MELIN, B.; JIMENEZ, C.; SAVOUREY, G.; BITTEL, J.; COTTET-EMARD, J.M.; PEQUIGNOT, J.M.; ALLEVARD, A.M.; GHARIB, C. Effects of hydration status and renal responses during moderate exercise in heat. European Journal Applied Physiology and Occupational Physiology, Berlin, v.76, n.4, p.320-7, 1997.

MOORE, J.; TIMPERIO, A.; CRAWFORD, D.; BURNS, C.; CAMERON-SMITH, D. Weight management and weight loss strategies of professional jockeys. International Journal of Sport Nutrition and Exercise Metabolism, Champaign. v.12, n.1, p.1-13, 2002.

MOUNTAIN, S.J.; COYLE, E.F. Influence of graded dehydration and hyperthermia in cardiovascular drift during exercise. Journal of Applied Physiology, Washington, v.73, n.4, p.1340-50, 1992.

NATIONAL COLLEGIATE ATHLETIC ASSOCIATION (NCAA). Wrestling rules and interpretations 2005. Disponível em: <http://www.ncaa.org>. Acesso em: 10 fev. 2005.

NYBO, L. CNS fatigue and prolonged exercise: effect of glucose supplementation. Medicine Science in Sports and Exercise, Madison, v.35, n.4, p.589-94, 2003.

OPPLIGER, R.A.; NELSON-STEEN, S.A.; SCOTT, J.R. Weight loss practices in college wrestlers. International Journal of Sport Nutrition and Exercise Metabolism, Champaign, v.13, n.1, p.29-46, 2003.

PERRIELLO, V.A. Aiming for healthy weight in wrestlers and other athletes. Contemporary Pediatrics, New Jersey, v.18, n.9, p.55-74, 2001.

ROCKWELL, M.; NICKOLS-RICHARDSON, S.; THYE, W. Nutrition knowledge, opinions, and practices of coaches and athletic trainers at a division I university. International Journal of Sport Nutrition and Exercise Metabolism, Champaign, v.11, n.2, p.174-85, 2001.

ROEMMICH, J.N.; SINNING, W.E. Weight loss and wrestling training: effect on nutrition, growth, maturation, body composition, and strength. Journal of Applied Physiology, Washington, v.82, n.6, p.1751-9, 1997.

SAWKA, M.N.; MONTAIN, S.J. Fluid electrolyte supplementation for exercise heat stress. The American Journal of Clinical Nutrition, Bethesda, v.72, p.564-72, 2000. Supplement.

SLAUGHTER, M.H.; LOHMAN, T.G.; BOILEAU, R.A.; HORSWILL, C.A.; STILLMAN, R.J.; VAN LOAN, M.D.; BEMBEN, D.A. Skinfold equations for estimation of body fatness in children and youth. Human Biology, Detroit, v.60, n.5, p.709-23, 1988.

SMITH, M.; DYSON, R.; HALE, T.; HAMILTON, M.; KELLY, J.; WELLINGTON, P. The effects of restricted energy and fluid intake on simulated amateur boxing performance. International Journal of Sport Nutrition and Exercise Metabolism, Champaign, v.11, n.2, p.238-47, 2001.

SOSSIN, K.; GIZIS, F.; MARQUART, L.; SOBAL, J. Nutrition beliefs, attitudes, and resource use of high school wrestling coaches. International Journal of Sport Nutrition and Exercise Metabolism, Champaign, v.7, n.3, p.219-28, 1997.

STEEN, N.S.; BROWNELL, K.D. Patterns of weight loss and regain in wrestlers: has the tradition change? Medicine Science in Sports and Exercise, Madison, v.22, n.6, p.762-8, 1990.

THORLAND, W.G.; TIPTON, C.M.; LOHMAN, T.G.; BOWERS, R.W.; HOUSH, T.J.; JOHNSON, G.O.; KELLY, J.M.; OPPLIGER, R.A.; TCHENG, T-K. Midwest wrestling study: prediction of minimal weight for high school wrestlers. Medicine Science in Sports and Exercise, Madison, v.23, n.9, p.1102-10, 1991.

VAN HALL, G.; SHIRREFFS, S.M.; CALBET, J.A.L. Muscle glycogen resynthesis during recovery from cycle exercise: no effect of additional protein ingestion. Journal of Applied Physiology, Bethesda, v.88, n.5, p.1631-6, 2000.

VANIN JUNIOR, R.; SAYLOR, K.E. Laxative abuse: a hazardous habit for weight control. Journal of American College Health, Washington, v.37, n.5, p.227-30, 1989. 
FABRINI, S.P. et al.

WORLD ANTI-DOPING AGENCY (WADA) . The 2005 prohibited list international standard: the World AntiDoping Code. 2005. Disponível em <http://www.wada-ama.org>. Acesso em: 24 fev. 2005.

ANEXO I - Questionário para quantificação de práticas de perda rápida de massa corporal em atletas.

UNIVERSIDADE FEDERAL DE VIÇOSA

DEPARTAMENTO DE NUTRIÇÃO E SAÚDE

Dados pessoais:

Nome: idade: sexo: M [ ] F [ ]

Tempo de prática na modalidade:

Antropometria:

Peso:

Estatura:

Dobras cutâneas

\begin{tabular}{|l|l|l|}
\hline Tríceps & Subescapular & Abdominal \\
\hline & & \\
\hline
\end{tabular}

1. Você tem o costume de pesar-se antes e depois dos treinamentos?

Sim [ ]

Não [ ]

2. Em média quantos dias antes da competição você inicia o controle da sua massa corporal?

3. Já utilizou de algum método para perda rápida de massa corporal? (< de uma semana para a competição) $\operatorname{Sim}[$ ] Não [ ]

4. Caso sua resposta seja afirmativa qual método utilizou?

[ ] aumento das atividades

[ ] restrição de líquidos

[ ] sauna/roupas antitranspirante

[ ] Dieta hipocalórica

[ ] restrição de lipídios

[ ] Restrição de carboidrados

[ ] uso de diuréticos e/ou laxantes

5. Ainda em relação à segunda questão, qual foi a maior redução de massa corporal apresentada na semana précompetitiva?

6. Caso você já tenha reduzido a massa corporal na semana antes da competição, quantos anos você tinha quando, pele primeira vez, reallizou este procedimento?

7. Já recebeu alguma orientação sobre como aumentar ou reduzir sua massa corporal? $\operatorname{Sim}[$ ]

Não [ ]

176 • Rev. bras. Educ. Fís. Esporte, São Paulo, v.24, n.2, p.165-77, abr./jun. 2010 
8. Em caso de orientação quem orientou?
[ ] médico
[ ] treinador
[ ] pais
[ ] livros
[ ] professor de educação física da escola

[ ] preparador físico

[ ] revistas

[ ] amigos

[ ] outros...

\section{Agradecimentos}

Aos atletas, senseis e diretores da Federação Mineira de Judô (FMJ) e da Liga Mineira de Judô (LMJ).

João Carlos Bouzas Laboratório de Performance Humana

Departamento de Educação Física Universidade Federal de Viçosa Av. P.H. Rolfs, s/n

36570-00o - Viçosa - MG - BRASIL e-mail: jcbouzas@ufv.br

Recebido para publicação: 07/01/2009

1a. Revisão: 18/06/2009

2a. Revisão: 08/10/2009

Aceito: 16/10/2009 